\title{
L'analyse du cycle de vie : un outil multicritères et quantitatif pour l'évaluation des impacts environnementaux
}

\author{
Angélique LÉOnARD, Sandra BELBOOM, Saïcha Gerbinet, \\ Sylvie Groslambert et Arribas MERCHAN \\ Chemical Engineering, ULiège
}

\section{Introduction}

De nombreuses méthodes ont vu le jour dans le but d'évaluer l'impact d'une activité ou d'un produit sur l'environnement. Certaines sont centrées sur les émissions de gaz à effet de serre comme Bilan Carbone ${ }^{\circledR}$ en France ou le Greenhouse Gas Protocol au niveau international. D'autres visent l'empreinte en eau comme Water Footprint. À côté de ces méthodes quantitatives et mono-critères, il existe des approches plus qualitatives, parmi lesquels l'affichage d'étiquettes énergétiques ou de labels à caractère " écologique ». Par rapport à ces approches, l'analyse du cycle vie (ACV) possède le double avantage d'être quantitative et multicritères, le potentiel de réchauffement climatique étant l'un des impacts évalués avec d'autres comme l'acidification, l'épuisement des ressources, l'utilisation d'eau, l'eutrophisation, la formation d'ozone photochimique, etc., ...

\section{Définition de l'analyse du cycle de vie}

L'analyse du cycle de vie (ACV) est une méthode standardisée par les normes ISO 14040 et 14044 (ISO, 2006a, ISO, 2006b). Elle traite des aspects et des impacts environnementaux potentiels tout au long du cycle de vie d'un produit. Ce cycle démarre à l'acquisition des matières premières et se termine par la fin de vie en passant par les étapes de production, d'utilisation, de transport et de recyclage. Le terme "produit» peut définir à la fois un produit au sens physique mais également un procédé ou un service. Cette méthode peut être appliquée du berceau à la tombe, du berceau à la porte (de l'usine) ou encore du berceau au berceau dans une approche d'économie circulaire.

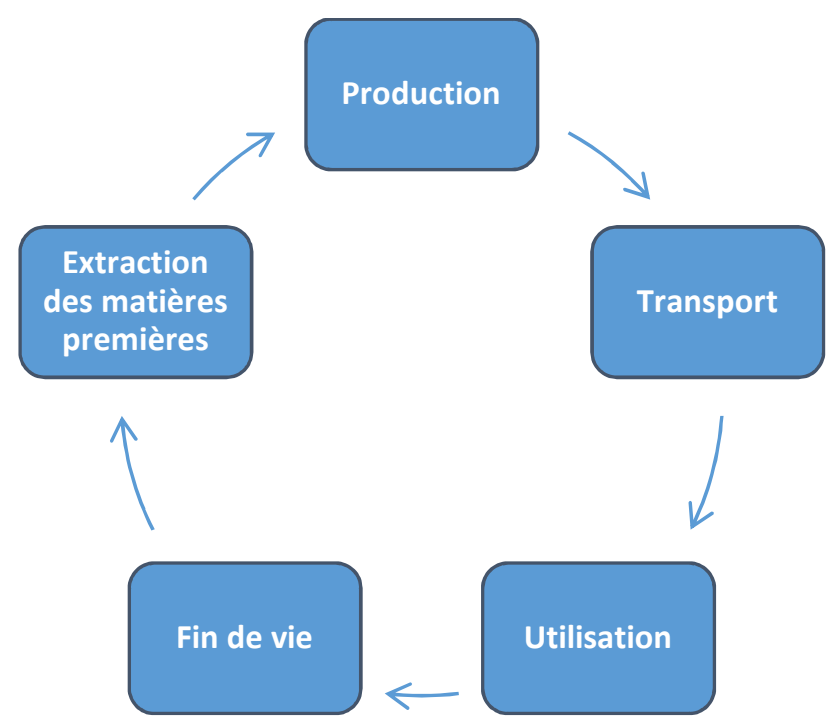

Figure 1 : Le cycle de vie d'un produit 


\section{Pourquoi une ACV ?}

L'ACV peut être menée à des fins de «diagnostic», permettant d'obtenir une vision des impacts environnementaux associés à un produit. Les résultats obtenus peuvent notamment être utilisés à des fins de communication environnementale, sous la forme de fiches de déclaration environnementale (écolabel de type III). L'ACV peut également être réalisée dans le cadre d'une démarche d'amélioration ou d'écoconception d'un produit. En effet, diverses solutions ou modifications peuvent être testées de manière 'théorique' afin d'accompagner des choix en recherche et développement visant à minimiser l'impact environnemental d'un produit ou d'un procédé de production au sens large. Les résultats issus d'une ACV peuvent également servir d'outil d'aide à la décision, dans le cadre d'achats durables par exemple ou encore de support au lancement de politiques publiques en lien avec l'environnement.

\section{Histoire que l'ACV}

La première étude d'ACV connue a été réalisée par Coca-Cola en 1969 pour déterminer l'intérêt, ou non, de fabriquer des cannettes métalliques. Le début des études ACV date donc de la fin des années 1960 et du début des années 1970 où les préoccupations étaient relatives à la consommation d'énergie et de matières premières ainsi qu'au traitement des déchets.

Au cours des années 1970 et 1980, le recours à l'ACV est resté limité en raison de la méconnaissance de la méthode. Son utilisation est restée confinée aux producteurs d'emballage ainsi qu'aux politiciens responsables de la gestion des déchets. Suite aux premiers chocs pétroliers, l'énergie était le point d'intérêt principal de ces études.

L'environnement et les conséquences des actions de l'homme sur ce dernier vont connaître un intérêt grandissant dans les années 1980 suite à des catastrophes chimiques comme l'accident de Bhopal en 1984 ou encore l'explosion nucléaire à Tchernobyl en 1986. Dès lors, la méthode de l'ACV restée relativement dans l'ombre jusqu'alors va susciter un regain d'intérêt. Cependant, l'ACV aboutissant à des résultats différents pour les mêmes produits est soumise à controverse et entraîne de nombreux débats (Baumann and Tillman, 2004, Astrup Jensen et al., 1997).

Dans les années 1990, la méthode va connaître une époque d'harmonisation où les différentes expériences et les acquis obtenus durant les deux décennies précédentes vont être mis en commun. La Société de Toxicologie et de Chimie Environnementale (SETAC) prend en main l'organisation de conférences et de groupes de travail pour l'amélioration de la méthode. Une société est également créée en 1992 (SPOLD : Society for the Promotion of Life Cycle Development) pour le développement de bases de données publiques et accessibles. Un code de conduite est publié en 1993 afin d'obtenir une harmonisation dans les étapes nécessaires à une telle étude. Des normes ISO sont ensuite développées en 1998 et remises à jour en 2006. La norme ISO 14040 définit les principes et le cadre de l'Analyse du Cycle de Vie, tandis que la norme 14044 regroupe le contenu technique nécessaire au bon déroulement d'une ACV. Dans le souci d'un cadrage encore plus précis, l'Union Européenne au travers du Joint Research Center a également publié un guide de bonnes pratiques relatif à la réalisation d'une ACV (Sustainability, 2010).

\section{Les quatre étapes d'une ACV}

Selon les standards ISO quatre étapes doivent être menées lors d'une ACV. Il s'agit de la description des objectifs et du champ de l'étude, de la réalisation de l'inventaire, de l'évaluation de l'impact et finalement de l'interprétation des résultats. Toutes ces étapes sont interdépendantes et le processus est itératif: des modifications peuvent être réalisées au cours du temps afin d'obtenir une cohérence entre les différentes étapes et d'affiner les résultats. 


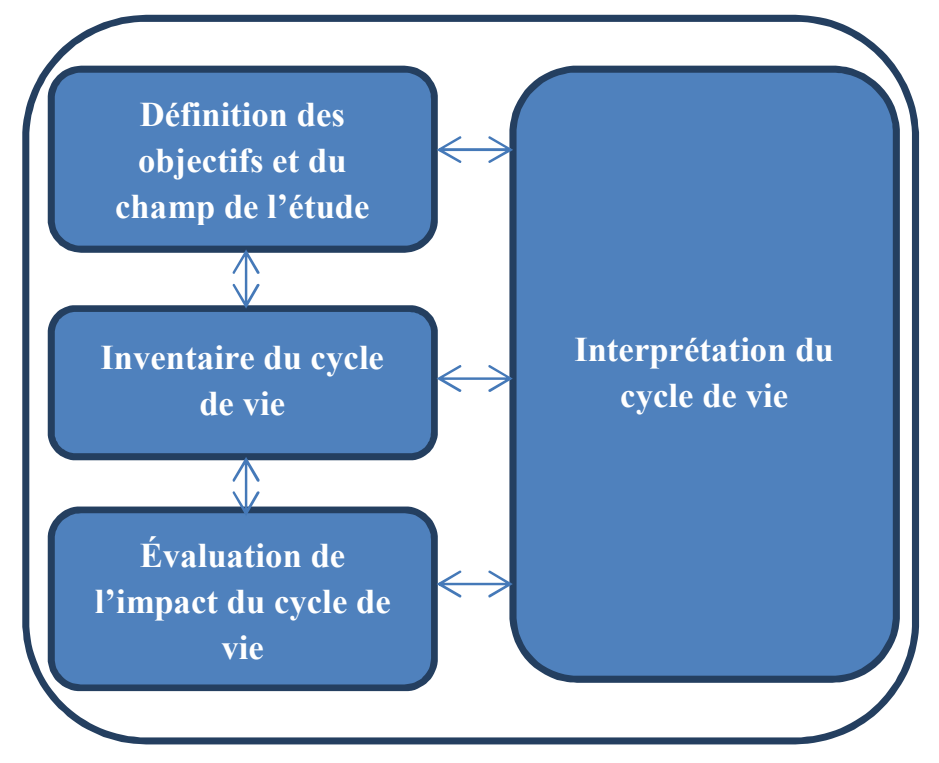

Figure 2. Les 4 étapes d'une ACV

\subsection{Définition des objectifs et du champ de l'étude}

Les objectifs doivent indiquer l'application envisagée suite à l'ACV, les raisons conduisant à réaliser l'étude ainsi que le public concerné. Le but de l'étude doit donc être défini de manière précise et ne pas se limiter à vouloir connaître les forces ou les faiblesses d'un produit. La précision est nécessaire pour effectuer les bons choix méthodologiques par la suite. Le but peut être exprimé par une question comme par exemple: «Quelles sont les possibilités d'amélioration dans le cycle de vie du produit étudié? », "Quelles sont les activités du cycle de vie qui contribuent le plus à l'impact environnemental du produit étudié ?», etc.

Le champ de l'étude doit décrire clairement des éléments tels que le système de produits à étudier, la fonction, l'unité fonctionnelle, les frontières, les règles d'allocation, la méthodologie, etc. La fonction est en lien avec les caractéristiques de performance du système étudié. L'unité fonctionnelle est définie comme l'unité de référence à laquelle les flux inclus dans les procédés doivent être reliés. Elle permet de normaliser les calculs ainsi que les bilans de matière et d'énergie réalisés. Les frontières du système déterminent les processus élémentaires qui sont pris en compte dans l'étude. Un processus élémentaire définit une étape du cycle de vie pour laquelle les données en entrée et sortie sont quantifiées.

Le choix de la méthode d'évaluation des impacts doit déjà être mentionné à ce stade de l'étude. Les normes ISO ne spécifient pas de méthode d'évaluation d'impact universelle mais demandent une justification de son utilisation ainsi que sa reconnaissance par le monde scientifique.

\subsection{Inventaire du cycle de vie}

Suite à la définition des objectifs et du champ de l'étude, les frontières du système sont connues ainsi que les données à collecter. Il s'agit de l'étape qui consomme le plus de temps car elle demande la collecte de tous les flux pertinents d'un point de vue environnemental, intervenant dans les processus élémentaires du système étudié (Figure 3). L'inventaire consiste donc en la réalisation des bilans de matière et d'énergie de tous les flux entrants et sortants pour chaque processus élémentaire. 


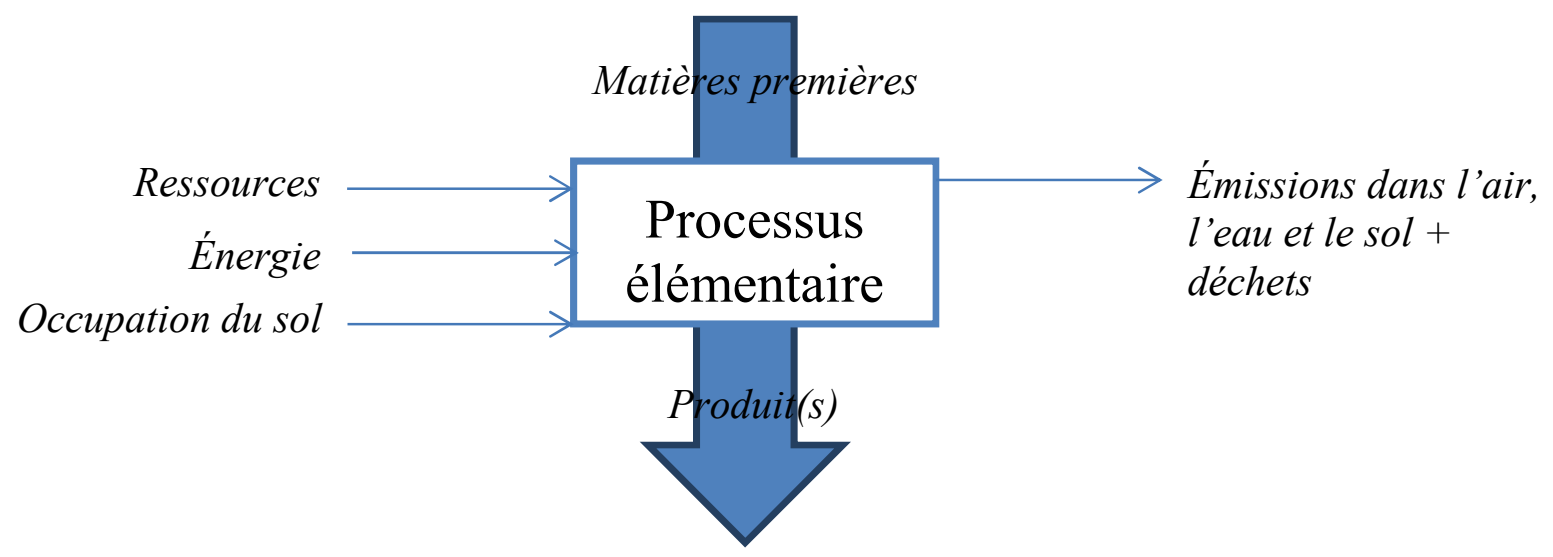

Figure 3 : Principe de l'inventaire sur un processus élémentaire

Il faut noter que la situation se complexifie dans le cas où un processus aboutit à plusieurs co-produits. Il sera parfois nécessaire de répartir l'inventaire, et en aval l'impact environnemental, entre ces divers co-produits. Différentes options d'allocation sont alors possibles, sur une base économique ou massique, par exemple.

En pratique cet inventaire repose à la fois sur la collecte de données primaires spécifiques au procédé étudié et sur des données génériques déjà disponibles dans des bases de données commerciales comme Ecoinvent ou Gabi. Par exemple, la donnée spécifique sera la consommation électrique d'un appareil et la donnée générique sera l'inventaire associé à la production d'électricité en fonction du mix électrique qui est pertinent.

\section{3 Évaluation de l'impact}

Une fois les données des bilans de matière et d'énergie obtenues pour chaque sous-processus du système étudié, l'impact environnemental peut être calculé. Cela consiste à décrire les conséquences sur l'environnement des émissions et des consommations obtenues lors de l'inventaire. Les standards imposent des étapes obligatoires lors de cette phase. Il s'agit de la définition des catégories d'impact, de la classification et de la caractérisation qui permet le calcul de l'impact environnemental par catégorie. La classification consiste à relier les substances émises ou consommées à des impacts environnementaux (par exemple tous les gaz à effet de serre vont être affectés à la catégorie de changement climatique). La caractérisation est la première étape permettant d'obtenir une réponse quantitative. Les impacts sont calculés par catégorie en utilisant des facteurs équivalents, appelés facteur de caractérisation, définis lors de la modélisation des chaînes de cause à effet. Par exemple, pour le changement climatique, les valeurs de Global Warming Potentiel à 100 ans fournis par le GIEC sont utilisées pour obtenir une réponse en $\mathrm{kg}_{\text {équivalent }} \mathrm{CO}_{2}$ tenant compte de l'ensemble des gaz à effet de serre émis. Ces facteurs de caractérisation peuvent dépendre de la méthode d'évaluation d'impact qui est utilisée, parmi lesquelles CML, ReCiPe, ILCD, ... Des étapes facultatives de normalisation, groupement ou encore de pondération peuvent également être menées lors de cette phase d'interprétation.

\subsection{Interprétation du cycle de vie}

La norme ISO 14040 (International Standardization Organization, 2006) définit l'interprétation du cycle de vie comme étant la phase au cours de laquelle les résultats obtenus lors de l'analyse de l'inventaire ou de l'impact sont combinés de manière consistante avec le but et le champ de l'étude afin d'obtenir des conclusions et des recommandations adéquates et pertinentes. Trois sous-étapes sont envisagées lors de l'interprétation du cycle de vie, à savoir l'identification des enjeux significatifs, la vérification de l'étude via des analyses d'incertitude et des études de sensibilité et la formulation des conclusions, limitations et recommandations liées à l'étude. 
Quelques cas d'études développés à l'ULiège vont être présentés de manière à illustrer le type d'information qui peut être obtenu via une ACV.

\section{Cas d'étude numéro 1 : polyéthylène classique vs. biopolyéthylène}

Durant sa thèse de doctorat (Belboom, 2013), Sandra Belboom a étudié l'impact environnemental de la production de bioéthanol à partir de canne à sucre, de betteraves sucrières et de blé. Dans un second temps, une comparaison a été effectuée entre le polyéthylène haute densité (PEHD) obtenu par la voie fossile classique (vapocraquage du naphta et polymérisation) et la voie agricole (via déshydratation catalytique de bioéthanol en éthylène et sa polymérisation). La figure 5 compare l'indicateur changement climatique pour le PEDH fossile et celui obtenu à partir de betteraves ou de canne à sucre sans tenir compte de changements d'affection des sols. Il est constaté que l'utilisation de matières premières agricoles permet de réduire les émissions de $\mathrm{CO}_{2 e ́ q}$ de 2000 à $3000 \mathrm{~kg}$ par tonne de $\mathrm{PEHD}$. Pour la canne à sucre la phase agricole est la plus

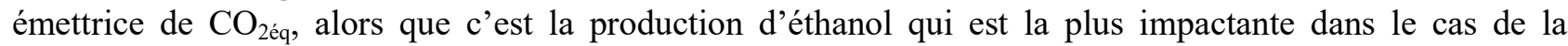
betterave. Cette différence est notamment due à la valorisation énergétique de la bagasse pour la filière canne à sucre, permettant de couvrir les besoins associés à la distillation.

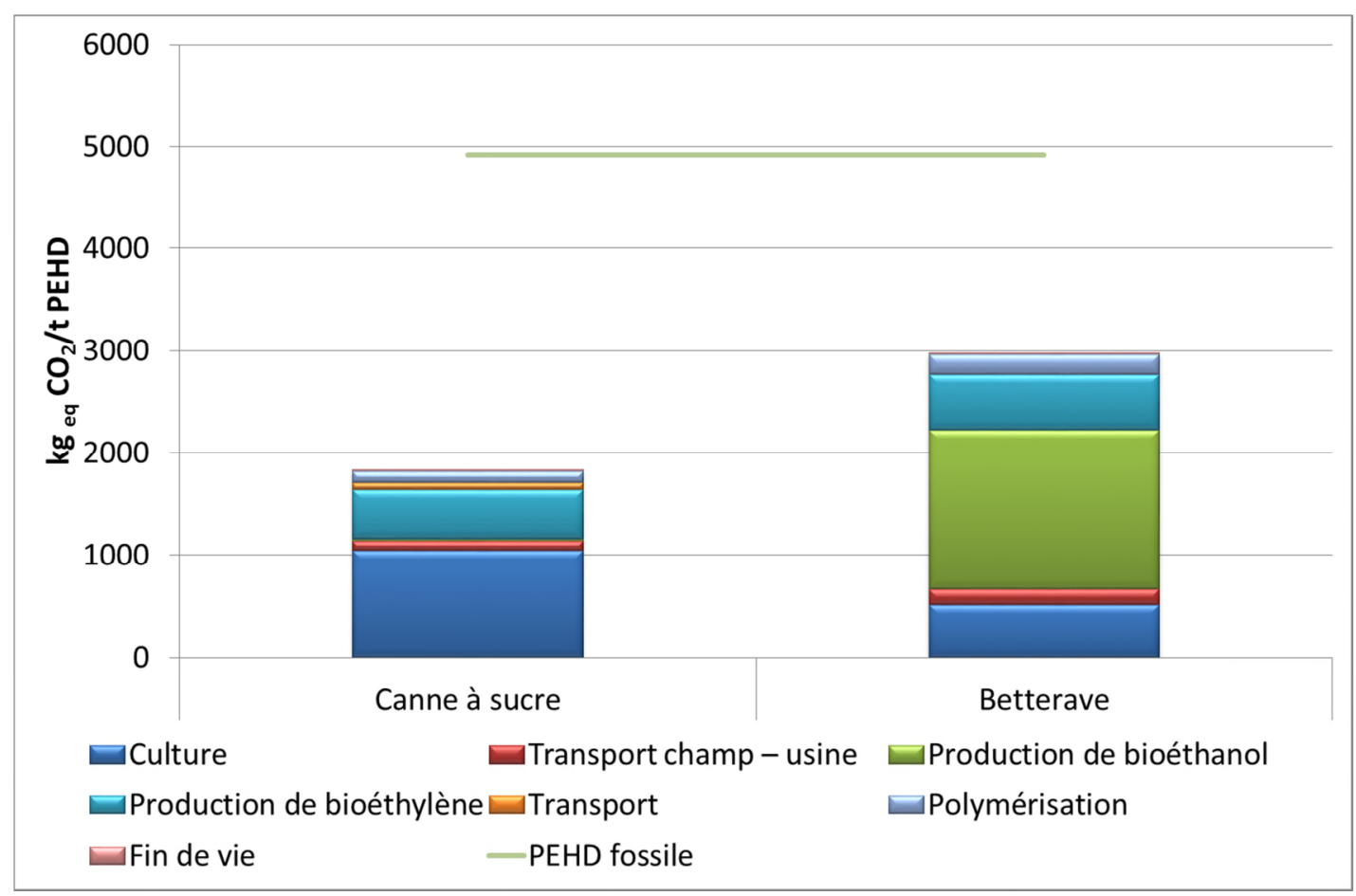

Figure 4 : Changement climatique pour le PEHD d'origine fossile ou biosourcé

La figure 5 illustre l'impact de divers scénarios tenant compte de changements direct (LUC = Land Use Change) ou indirect (ILUC = Indirect Land Use Change) d'affectation des sols. En effet, le recours massif à la canne à sucre nécessiterait une extension des surfaces cultivées, au détriment des pâturages. Ce changement direct d'affectation mène à une libération d'une partie du carbone stocké dans le sol. De plus, si on considère une compensation partielle ou totale des pâturages perdus via de la déforestation, de nouvelles émissions de $\mathrm{CO}_{2}$ sont à prendre en compte, selon le concept de changement indirect d'affectation des sols. Dans le cas de la prise en compte de changements indirects associés à la déforestation, le PEHD d'origine fossile est moins impactant du point de vue changement climatique que la version agrosourcée à base de canne à sucre. Plus de détails sont disponibles dans les publications issues de la thèse (Belboom and Léonard, 2014, Belboom and Léonard, 2016) 
Bulletin de la Société Royale des Sciences, Vol. 88, Actes de colloques, 2019, p. 73 - 81

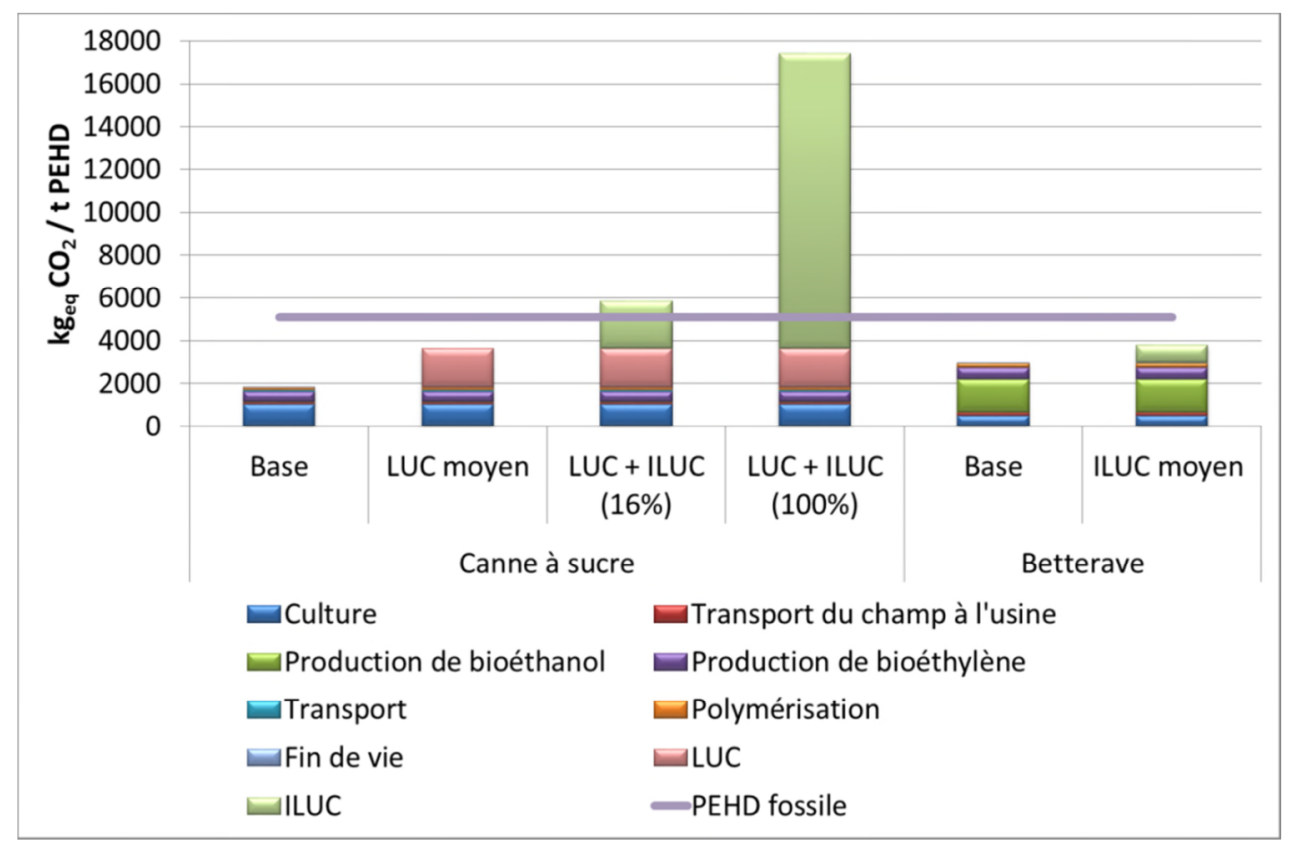

Figure 5 : Effet de la prise en compte de changements d'affectation des sols sur le changement climatique

\section{Cas d'étude numéro 2 : transport de marchandises par voie multimodale}

Angel Merchan a étudié l'impact environnemental associé au transport de marchandise par voie multimodale dans le contexte du projet collaboratif Brain-Trains financé par Belspo (Merchan Arribas, 2019). Un des résultats majeurs est l'importance du mix électrique d'une part, et du taux de chargement et de la performance environnementale des camions d'autre part. La Figure 6 compare divers impacts environnementaux pour le transport d'une tonne de marchandise entre Anvers et Ludwigshaufen pour divers modes de transport. Si on se focalise sur le changement climatique, les résultats montrent que le moyen de transport le moins émetteur est un camion (34 à 40 tonnes) avec un taux de chargement de $85 \%$ et de technologie EURO V. Le transport via train à moteur électrique est le plus impactant, en lien avec le mix électrique allemand. La voie d'eau (IWW) se positionne de manière intermédiaire, un des facteurs clés étant la distance parcourue en fonction du positionnement de ces voies.

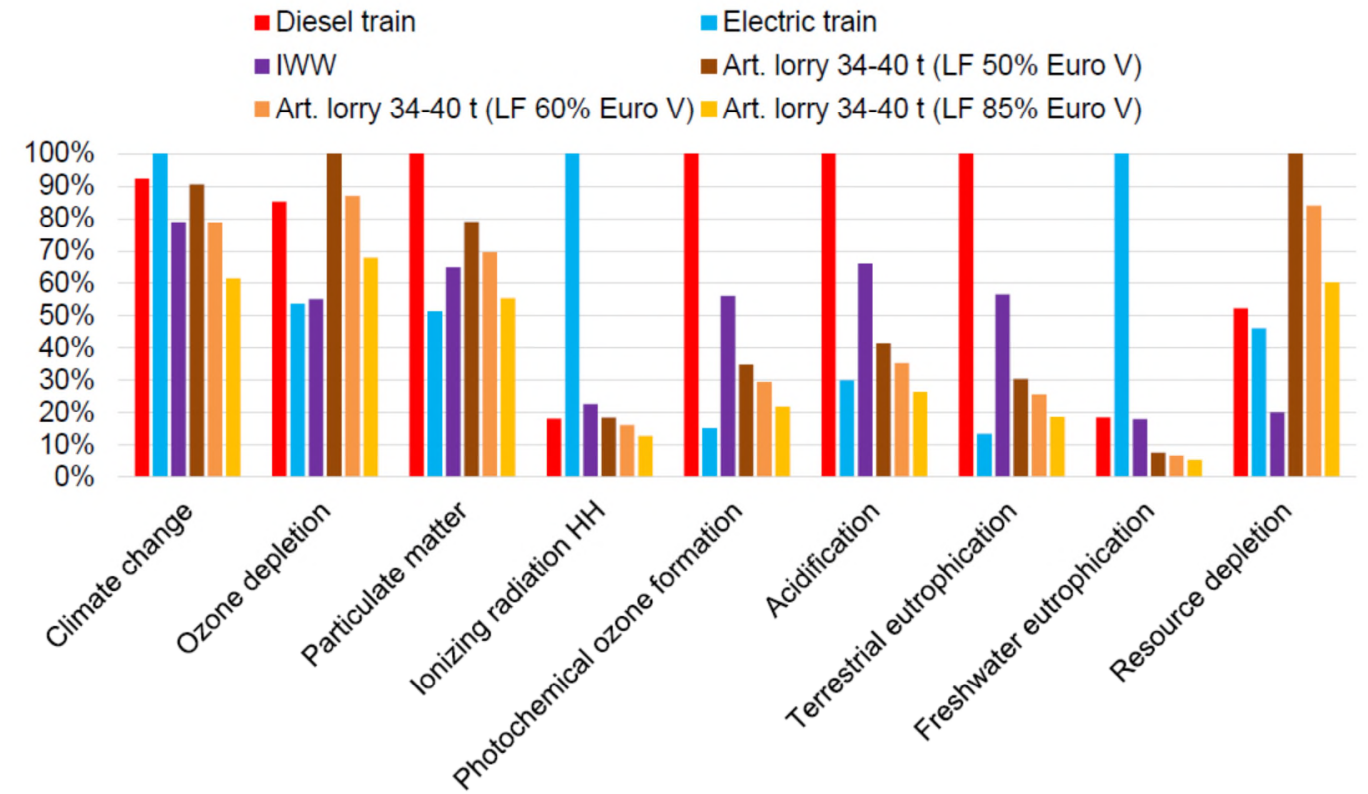

Figure 6. Impact environnemental du transport de 1 tonne de marchandise d'Anvers à Ludwigshaufen - ReCiPe 


\section{Cas d'étude numéro 3: impact de l'incorporation de matières recyclées et de l'allègement}

La Figure 7 illustre l'évolution de l'impact environnemental de la bouteille en PET de 1,5 litres commercialisée par Spadel. Au cours des années, l'allègement de la bouteille d'une part (de 51 à $28,5 \mathrm{~g}$ ) et l'incorporation de PET recyclé d'autre part ont permis une réduction de plus de $60 \%$ de l'indicateur de changement climatique.

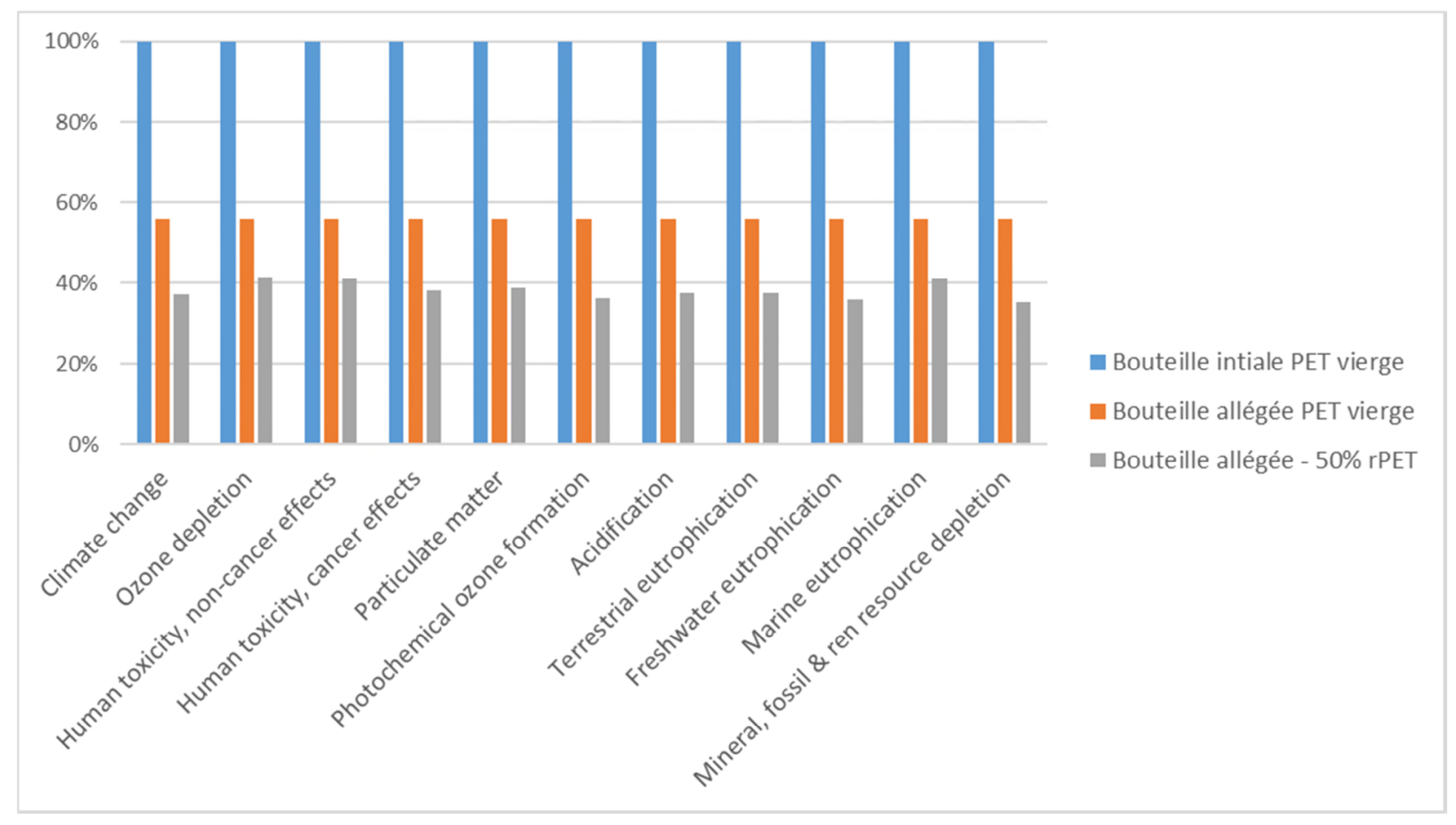

Figure 7. Effet de l'incorporation de matières recyclées et de l'allègement sur l'impact environnemental de bouteilles - ILCD

\section{Cas d'étude numéro 4 : agriculture conventionnelle et biologique}

La Figure 8 est issue des travaux de thèse de Saicha Gerbinet (Gerbinet, 2018). Elle compare la culture conventionnelle et la culture biologique de blé en Belgique. Concernant le changement climatique, et sur une base massique, les résultats montrent que le changement climatique est $20 \%$ plus bas pour la culture conventionnelle. Ce résultat a priori interpellant est notamment lié aux rendements plus faibles de la culture biologique et aux émissions aux champs qui sont plus importantes dans le cas d'engrais naturels de type fumier ou lisier. 


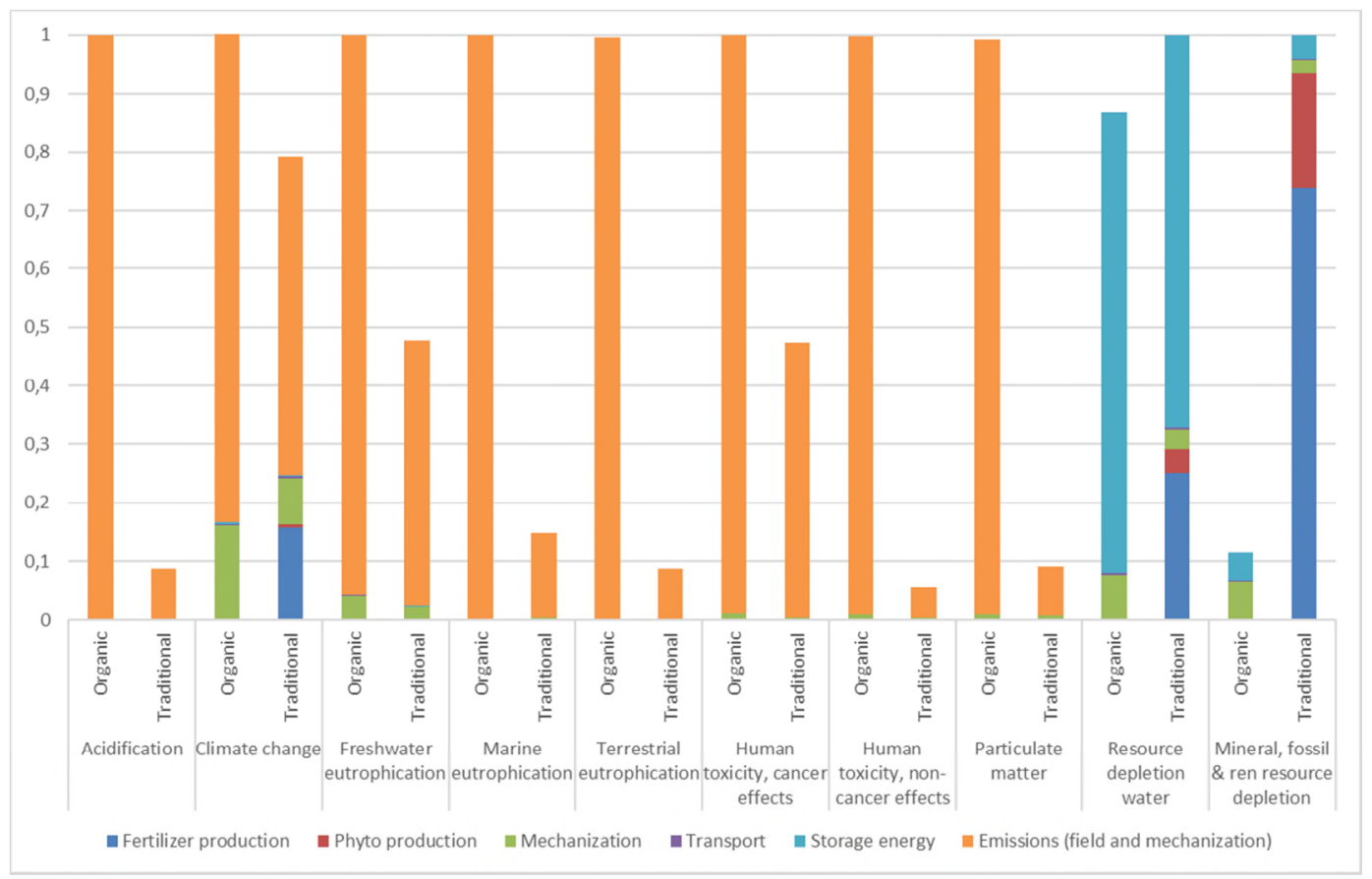

Figure 8. Blé en culture conventionnelle vs. culture biologique - ILCD - Sur base massique

\section{Conclusions}

L'ACV est devenue une méthode de choix pour l'évaluation de l'impact environnemental d'un produit. Cette méthodologie reste toutefois assez complexe à mettre en œuvre et demande un certain niveau de connaissance des outils et des méthodologies d'évaluation de l'impact. Le risque de mauvaise interprétation et de raccourcis reste possible et un regard critique est nécessaire lorsque des résultats d'ACV sont présentés. La publication d'études transparentes et critiques devrait permettre dans le futur de renforcer la crédibilité de cet outil et d'en favoriser un emploi encore plus large.

\section{Références}

Astrup Jensen, A., Hoffman, L., Moller, B. T., Schmidt, A., Christiansen, K., Elkington, J. \& Van Dijk, F. 1997. Life Cycle Assessment - A guide to approaches, experiences and information sources. In: EUROPEAN ENVIRONMENT ENERGY (ed.).

Baumann, H. \& Tillman, A.-M. 2004. Development of the LCA concept. In: STUDENTLITTERATUR (ed.) The Hitch Hiker's Guide to LCA. Lund, Sweden: Studentlitteratur AB.

Belboom, S. 2013. Evaluation de l'impact environnemental de la production de bioéthanol à partir de canne $\grave{a}$ sucre, betterave ou froment par analyse du cycle de vie. Comparaison des utilisations biocarburant et bioplastique. $\mathrm{PhD}$ Thesis, University of Liège.

Belboom, S. \& Léonard, A. 2014. Importance of LUC and ILUC on the carbon footprint of bioproduct: case of bio-HDPE. Matériaux et Techniques, 102.

Belboom, S. \& Léonard, A. 2016. Does biobased polymer achieve better environmental impacts than fossil polymer? Comparison of fossil HDPE and biobased HDPE produced from sugar beet and wheat. Biomass and Bioenergy, 85, 159-167.

Gerbinet, S. 2018. LCA of building materials that include a biobased binder : lessons and challenges. $\mathrm{PhD}$ thesis, University of Liège.

International Standardization Organization 2006. ISO 14040 : Management environnemental - Analyse du cycle de vie - Principes et cadre. ISO.

Iso 2006a. ISO 14040 : Management environnemental - Analyse du cycle de vie - Principes et cadre, ISO.

Iso 2006b. ISO 14044 : Management environnemental - Analyse du cycle de vie - Exigences et lignes directrices, ISO. 
Bulletin de la Société Royale des Sciences, Vol. 88, Actes de colloques, 2019, p. 73 - 81

Merchan Arribas, A. 2019. Environmental impact assessment of rail freight intermodality in Belgium using a Life Cycle Assessment approach. $\mathrm{PhD}$ thesis, University of Liège.

Sustainability, J. R. C.-I. F. E. A. 2010. General guide for Life Cycle Assessment - ILCD Handbook, Publications Office of the European Union. 